\title{
Estimativa da partição de energia na superfície
}

\section{Estimate of the surface energy partition from air temperature and specific humidity meteorological time series}

\author{
Daiane de Vargas Brondani, Otávio Costa Acevedo, Fabíola Valente \\ Universidade Federal de Santa Maria - RS, Brasil \\ meteorologia.daia@gmail.com; otavio@ufsm.br; fabiola_krolina@hotmail.com
}

\begin{abstract}
Resumo
Este trabalho é uma análise complementar ao método em desenvolvimento, que propõe estimar a partição de energia na superfície pelo método da razão de Bowen e a altura da camada limite convectiva na escala mensal baseando-se na evolução temporal média das variáveis temperatura e umidade específica do ar. A hipótese básica é que a evolução dessas quantidades é controlada unicamente pela convergência dos fluxos superficiais de calor sensível e calor latente. Essa suposição é válida para escala mensal e em regiões de latitudes médias afastadas da costa. Dessa forma, presume-se que os termos advectivos, da equação de balanço dessas quantidades na camada limite convectiva, nas situações pré-frontais e pós-frontais apresentam sinal oposto. Assim, utilizando-se para uma escala temporal mais longa que a escala típica da passagem dos sistemas sinóticos, a hipótese de cancelamento dos termos pode ser testada. Inicialmente, o método é aplicado para a região de Santa Maria, onde é assumido que as condições que permitem desprezar os termos advectivos em escala mensal são válidas. Ao testar o método em diferentes escalas temporais: $5,10,15,20$ e 30 dias, os menores erros de temperatura e umidade específica do ar foram para 15 e 30 dias, enquanto que os fluxos de calor sensivel e de calor latente apresentaram menores erros relativos em 20 e 30 dias,respectivamente.
\end{abstract}

Palavras-chave: Fluxos superficiais, altura da camada limite convectiva, temperatura do ar, umidade específica do ar.

\begin{abstract}
This paper is a complementary analysis to method development, which proposes to estimate the energy partition on the surface by Bowen ratio method and height of convective boundary layer on the monthly scale based on the average temporal evolution of the variables air temperature and specific humidity. The basic hypothesis is that the evolution of these quantities is controlled solely by the convergence of surface fluxes of sensible and latent heat. This assumption is valid for monthly scale and in regions of middle latitudes away from the coast. Thus, it is assumed that the advective terms of the balance equation of these quantities in the convective boundary layer, the prefrontal situations and post-frontal have opposite sign. Therefore, using for a longer time scale than the typical scale of the passage of synoptic systems, the cancellation terms of hypothesis can be tested. In this study, the method is applied to the region of Santa Maria, where it is assumed that the conditions allowing despise the advective terms in monthly scale are valid. When testing the method on different time scales: 5,10,15,20 and 30 days, smaller errors temperature and specific humidity were for 15 and 30 days, while the sensible heat and latent heat fluxes showed lower relative errors at 20 and 30 days, respectively.
\end{abstract}

Keywords: Surface fluxes, height of convective boundary layer, air temperature,specific humidity. 


\section{Introdução}

Os fluxos turbulentos de calor sensível $(H)$ e latente $(L E)$ são os principais constituintes do balanço de energia no sistema terra-atmosfera e atuam no transporte das quantidades, temperatura $(T)$ e umidade específica do ar $(q)$ ao longo da camada limite atmosférica (CLA).

Após o nascer do sol, quando a energia radiativa disponível $\left(Q^{*}\right)$ passa a ser positiva, um regime convectivo é observado (Sorbjan, 2007), característica principal da camada limite convectiva (CLC), então formada. Nessa fase, CLC coexiste com a camada limite noturna (CLN), a qual inicialmente confere maior resistência ao movimento convectivo. Com o aumento de $Q^{*}$ ocorre um aumento de $H$ e as termas convectivas penetram na CLN até o ponto dessa camada ser totalmente erodida. Vencida a CLN, a CLC cresce rapidamente devido à estratificação neutra da camada residual (CR). Assim como $H$ atua para redistribuir a temperatura verticalmente na CLA, LE homogeneíza os diferentes conteúdos de umidade na CLA fazendo com que essas quantidades não se acumulem na superfície.

Além disso, $H$ e $L E$ contribuem para identificar mudanças climáticas; no setor energético ou industrial, possibilitam a otimização do uso da energia (Brutsaert, 2013) e em modelos hidrológicos, meteorológicos ou agronômicos permitem melhorias no planejamento e gerenciamento de recursos hídricos (Bastiaanssen et al., 1998), entre outras aplicações. Contudo, a estimativa desses fluxos não é trivial. Os métodos para a determinação de $H$ e $L E$ variam entre os métodos de estimativa local e que necessitam de recursos financeiros apreciáveis para sua instalação e manutenção, como o método da covariância dos vórtices turbulentos (do termo em inglês eddy covariance) (Stull, 1988) até métodos mais simples, como o método da razão de Bowen, em que relaciona os fluxos $H$ e $L E$ e métodos de estimativa regional como o proposto por Bastiaanssen et al. (1998).

Há um trabalho em desenvolvimento com o objetivo de estimar $H$ e $L E$ baseado em proposições simples e que utilizam dados de temperatura e umidade específica do ar das 9 h às 16 h. A escala temporal adotada é a mensal, devido à suposição de que na média para um dado mês, a evolução temporal de $T$ e $q$ depende unicamente dos fluxos de $H$ e $L E$ e da sua convergência na CLC. Contudo, não havia sido verificado, de forma objetiva, a escala temporal adequada para aplicação do método. Com isso, o objetivo do trabalho é analisar o efeito de diferentes escalas temporais sobre os erros de $T$ e $q$ e dos fluxos de $H$ e $L E$

\section{Metodologia}

A hipótese básica do método é de que as evoluções temporais médias das variáveis $T$ e $q$ são controladas unicamente pelos fluxos superficiais de $H$ e $L E$ e sua convergência na CLC (Fitzjarrald et al., 2001). O método é aplicado em Santa Maria que está situada em latitudes médias, onde se pode esperar que na escala mensal os termos advectivos apresentem sinais opostos entre as situações pré-frontais e pós-frontais, e assim, tornandose nulas, além do que é um local afastado da costa, o que reduz a possibilidade de haver advecção média de $T$ e $q$. Com isso, as equações do balanço para $T$ e $q$, na escala mensal são reduzidas a:

$$
\begin{aligned}
& h \frac{\partial \bar{q}}{\partial t}=\left(\overline{w^{\prime} q^{\prime}}\right)_{0}-\left(\overline{w^{\prime} q^{\prime}}\right)_{h}=\left(\overline{w^{\prime} q^{\prime}}\right)_{0} \\
& h \frac{\partial \bar{\theta}}{\partial t}=\left(\overline{w^{\prime} \theta^{\prime}}\right)_{0}-\left(\overline{w^{\prime} \theta^{\prime}}\right)_{h}=\left(\overline{w^{\prime} \theta^{\prime}}\right)_{0}
\end{aligned}
$$

Nas Equações 1 e 2, os fluxos de $H$ e $L E$ no topo da CLC, $h$, são aproximadamente zero devido à transição entre o regime turbulento na CLC para a atmosfera livre, onde a turbulência inexiste (Stull, 1988). As variáveis médias $T$ e $q$ são obtidas a partir da estação do INMET e a altura, $h$, entre 9 h01min e $16 \mathrm{~h}$ foi determinada pelo método termodinâmico (Stull, 1988), em que foi imposto um fluxo cinemático de $H$ e utilizado o perfil da radiossondagem das $12 Z$, conforme pode ser visualizado nas Figuras 1(a) e 1(b).

O primeiro valor de $h$ é obtido iterativamente variando de 100 a $1000 \mathrm{~m}$, em intervalos de $50 \mathrm{~m}$. Os próximos valores são selecionados a partir da menor diferença entre as áreas de 1(a) e 1(b) que correspondem às energias 1 e 2 das equações 3 e 4 , respectivamente, ou seja a energia necessária no crescimento é proveniente de $H_{c}$.

$$
E_{1}=H_{c[n-1]} \cdot \Delta t+\frac{\left(H_{c[n]}-H_{c[n-1]}\right) \cdot \Delta t}{2}
$$

sendo que o índice $n$ se refere ao tempo final e o índice $[n-1]$ é o tempo inicial, sendo esses tempos separados por $\Delta t$, que é o tempo de integração e se assume ser de $60 \mathrm{~s}$.

$$
E_{2}=h_{[n-1]} \cdot \Delta \theta+\frac{\left(h_{[n]}-h_{[n-1]}\right) \cdot \Delta \theta}{2}
$$

em que $\Delta \theta$ é a diferença entre $\theta$ no tempo final e inicial do intervalo.

A estimativa de $H_{c}$ foi realizada da seguinte forma:

$$
H=\frac{Q^{*}}{\left(1+\frac{1}{\beta}\right)}
$$

em que $Q^{*}$ é uma expressão matemática senoidal dependente do saldo de radiação horário máximo mensal, 


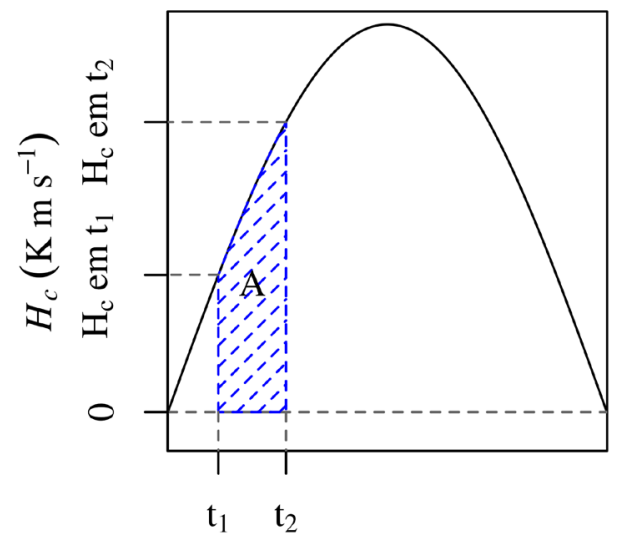

Tempo

(a)

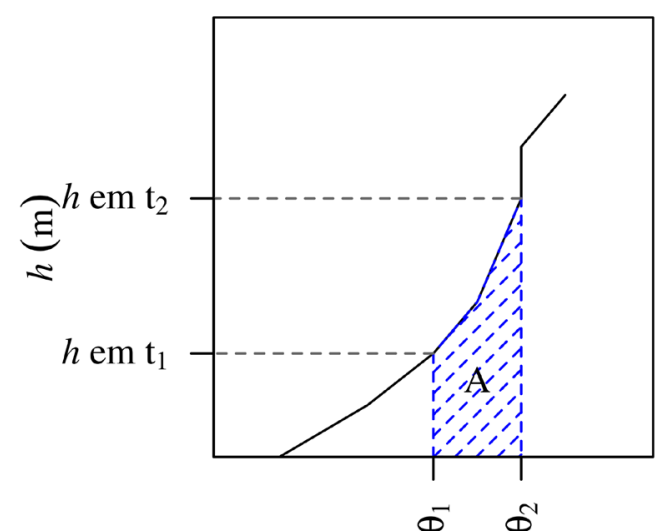

$\theta(\mathrm{K})$

(b)

Figura 1: (a) Variação idealizada do fluxo cinemático de calor sensível com condição de céu claro sobre continente. A área hachurada em azul representa a quantidade de entrada de energia,na forma de calor sensível, na CLC, após o nascer do sol entre os tempos $t_{1}$ e $t_{2}$. (b) Radiossondagem típica da manhã, exibindo a variação de $\theta$ com $h$ entre o intervalo $t_{1}$ e $t_{2}$. As áreas hachuradas em (a) e (b) são iguais. [Figura adaptada de Stull (1988)]

$Q_{\max }^{*}$, e do comprimento astronômico do dia $N$, o qual é o número de minutos em que o saldo de radiação é positivo:

$$
Q^{*}=Q_{\max }^{*} \operatorname{sen}\left\{\frac{\left[t-\left(12-\frac{N}{2}\right)\right] \pi}{N}\right\}
$$

no qual $Q_{\text {max }}^{*}$ refere-se aos valores máximos médios men- sais obtidos a partir da estação micrometeorológica. O valor de $N$ é resultado da interpolação linear para verificar qual intervalo de tempo corresponde a $Q^{*}$ positivo.

\subsection{Evolução da CLC e relações para $q$ e $T$}

A CLC responde rapidamente às forçantes da superfície, o que resulta num claro ciclo diurno. Logo após o nascer do sol, quando $Q^{*}$ passa a ser positivo, a marcada instabilidade impulsiona mistura vertical e turbulência. Neste momento, a CLC, CN e a CR coexistem. À medida que o tempo evolui, mais energia $H$ é transferida verticalmente, a expansão da CLC aumenta e a CN e CR são erodidas totalmente. Assim, conforme CLC avança sobre as duas camadas, características como $T$ e $q$ são incorporadas a esta camada. Matematicamente, desconsiderando-se os processos advectivos, esta relação entre $h, H$ e $T$, é quantificada pela equação:

$$
T_{[n]}=\frac{T_{[n-1]} \cdot h_{[n-1]}+\bar{T} \cdot\left(h_{[n]}-h_{[n-1]}\right)}{h_{[n]}}+\left(\frac{H_{[n]}}{h_{[n]}}\right) \cdot 60
$$

De modo análogo, a superfície também fornece $L E$ à CLC e o avanço dessa camada em direção às camadas $\mathrm{CN}$ e CR, permite adição de $q$. Assim, a equação para $q$ é obtida por:

$q_{[n]}=\frac{q_{[n-1]} \cdot h[n-1]+\bar{q} \cdot\left(h_{[n]}-h_{[n-1]}\right)}{h_{[n]}}+\left(\frac{L E_{[n]}}{h_{[n]}}\right) \cdot 60$

Nas equações 7 e $8, T_{[n]}$ e $q_{[n]}$, onde $n=\{2,420\}$, são as estimativas para as variáveis $T$ e $q$ na altura $h_{[n]} \cdot \bar{q} \mathrm{e}$ $\bar{T}$ são os valores médios das variáveis $T$ e $q$ adicionadas pelo crescimento da CLC à medida que ocorre o passo de tempo de um minuto e são obtidas da radiossondagem. $T$ e $q$ no instante $n=1$ correspondem às médias horárias mensais das observações às $9 \mathrm{~h}$, da estação do INMET. $H$ é obtido através da equação 5 e a variável de entrada, $Q^{*}$, determinada pela equação 6 . $L E$ é quantificado pela equação: $L E=\frac{H}{\beta}$. $\beta$ em $H$ e $L E$ é obtido iterativamente, assim como $h_{1}$ (no texto convencionou-se chamar de $h_{0}$ e daqui para frente será assim referenciado). O primeiro termo do lado direito nas equações 7 e 8 , representam o resultado da erosão da CLC na camada acima conforme ocorre seu crescimento e o segundo termo expressa a contribuição devido a $H$ e $L E$ distribuídos em $h$, nessa camada.

A evolução de $h_{[n]}$, baseado no método termodinâmico, é inicialmente realizada com a atribuição da altura inicial, $h_{0}$, que equivale a $100 \mathrm{~m}$. O aumento de $h_{[n]}$ ocorre à medida que mais energia fornecida por $H$ é absorvida pela CLC. A seleção de $h_{[n]}$ para cada minuto é realizada verificando-se a menor diferença entre as 
energias 1 (da curva de $H$ imposto) e 2 (da radiossondagem). Realizada a primeira execução do método com $h_{0}=100 \mathrm{~m}$ e $\beta$ variando de 0 a 2 com passos de 0,01 , o algoritmo passa para $h_{0}=150 \mathrm{~m}$ e determina a evolução de $h_{[n]}$ novamente, $\operatorname{com} \beta$ variante. Isso é realizado até todos valores de $h_{0}$ terem sido simulados. Com essas simulações, o método verifica a menor soma da raiz do erro quadrático médio (do termo em inglês root mean square error (RMSE)) entre $T$ e $q$, a qual está associada ao valor de $\beta$ e $h_{0}$ selecionados para um dado mês.

\subsection{Determinação de $\beta$ e $h_{0}$}

As variáveis $\beta$ e $h_{0}$ são parâmetros livres no presente modelo. Assim, são atribuídos valores a elas, de 0 a 2 com passos de 0,01 para $\beta$ e de 100 a $1000 \mathrm{~m} \mathrm{em}$ intervalos de $50 \mathrm{~m}$ para $h_{0}$. O modelo seleciona os valores de $\beta$ e $h_{0}$ a partir da menor soma de RMSE entre $T$ e $q$. As equações 9 e 10 representam o RMSE para as variáveis $T$ e $q$, respectivamente:

$$
\begin{aligned}
& \operatorname{RMSE}_{T}=\left[\frac{1}{N} \sum_{n=1}^{N}\left(T_{\text {nest }}-T_{\text {nobs }}\right)^{2}\right]^{\frac{1}{2}} \\
& \operatorname{RMSE}_{q}=\left[\frac{1}{N} \sum_{n=1}^{N}\left(q_{\text {nest }}-q_{\text {nobs }}\right)^{2}\right]^{\frac{1}{2}}
\end{aligned}
$$

Nas equações 9 e 10, $N$ corresponde ao número de minutos, variando de $n=1$ a $N=420 \mathrm{~min}$. $T_{\text {nest }}$ e $q_{\text {nest }}$ referem-se às estimativas de $T$ e $q$ pelo método proposto, enquanto que $T_{\text {nobs }}$ e $q_{\text {nobs }}$ correspondem às observações dessas variáveis obtidas pelo INMET.

\subsection{Avaliação dos erros}

Cada mês foi dividido em escalas temporais de 5,10,15,20 e 30 dias. Essa última escala foi assim nomeada e se refere ao mês cheio. A partir de um mês qualquer, como por exemplo, janeiro, contou-se de trás para frente, a partir do dia 31, as escalas 20,15,10 e 5 e executou-se o método. Com isso, para cada execução, foram obtidos valores dos erros de $T$ e $q$, estimativas de $H$ e $L E$ que foram comparados com os valores observados. Foram feitas médias aritméticas dos erros de $T$ e $q$ para cada escala. Além disso, determinou-se o erro relativo para $H$ e $L E$, respectivamente, conforme as equações e .

$$
\begin{gathered}
\left|\frac{\bar{H}_{o b s}-\bar{H}_{e s t}}{\bar{H}_{o b s}}\right| \\
\left|\frac{\bar{L} \bar{E}_{o b s}-\overline{L E}_{e s t}}{L E_{o b s}}\right|
\end{gathered}
$$

em que $\bar{H}_{o b s}$ e $\overline{L E}_{\text {obs }}$ referem-se aos valores médios observados de $H$ e $L E$, respectivamente e $\bar{H}_{\text {est }}$ e $\overline{L E}_{\text {est }}$ são as estimativas médias para $H$ e $L E$, nessa ordem.

\subsection{Dados observacionais}

Foram utilizadas variáveis meteorológicas do período entre novembro de 2013 e novembro de 2014 para Santa Maria (Latitude: 29.7 S, Longitude: 53.7 O, Altitude: 103 $\mathrm{m})$ das $9 \mathrm{~h}$ às $16 \mathrm{~h}$ local, referente às medidas médias horárias de $T, q$, obtidas pelo INMET. Além dessas medidas, foram utilizadas $T$ e $q$ médias de radiossondagens atmosféricas das 12Z, lançadas na BASM (Latitude: 29,71 S, Longitude: 53,69 O, Altitude: $85 \mathrm{~m}$ ).

Para comparar os fluxos de $H$ e $L E$ observados e estimados utilizou-se um conjunto de dados obtido da Torre Micrometeorológica da rede SULFLUX, localizada em área pertencente à Universidade Federal de Santa Maria - UFSM (Latitude: -29,7241; Longitude: -53,7597; Altitude: $88 \mathrm{~m}$ ). A região é coberta por campo nativo do bioma Pampa, vegetação típica do estado gaúcho. A vegetação campestre dominante da Depressão Central do estado é caracterizada por dois tipos de estratos: o mais baixo, formado por gramíneas rizomatosas, e o mais alto, composto por touceiras de gramíneas cespitosas e, ainda por espécies de compostas (Veldman et al., 2015). O clima de Santa Maria, de acordo com a classificação de Köppen é do tipo Cfa, chuvoso e úmido, com temperatura anual média de 19,5 C, sendo a mínima registrada nos meses de julho e agosto, estação do inverno. A precipitação anual média é aproximadamente $1650 \mathrm{~mm}$. A torre micrometeorológica é composta por um Sistema Eddy Covariance (EC), instalado a 3 metros de altura, consistindo de um Analisador de Gás Infravermelho de caminho fechado (LI 7200, LICOR) e um anemômetro sônico 3D (Wind Master Pro, Gill Instruments), com medições realizadas em frequência de $10 \mathrm{~Hz}$. As componentes radiativas foram medidas em baixa frequência ( 1 minuto) por um saldo radiômetro (CNR4, Kipp Zonnen), posicionado também a 3 metros e foram calculadas para resultar em $Q^{*}$. Posteriormente, interpolou-se linearmente essa variável para verificar quantas horas correspondiam a valores positivos de $Q^{*}$, o que resultou em $N$. Os dados brutos do sistema Eddy Covariance foram processados por um software comercial (EddyProß), V 5.1.1 - Li-Cor, Lincon, Nebraska, USA). Para o cálculo e correção dos fluxos de energia e massa foi utilizada uma configuração padrão do software: flutuações turbulentas em torno de médias por bloco de 30 minutos, flutuações de densidade (Webb et al., 1980), rotação de coordenadas dupla (Wilczak et al., 2001), correção do ângulo de ataque para as componentes do vento (Nakai e Shimoyama, 2012), maximização da covariância (padrão) para compensação da defasagem de tempo entre os sensores, filtro passa alta e baixa (Moncrieff et al., 1997), testes de controle de qualidade dos fluxos (Foken et al., 2004). 


\section{Resultados e discussões}

\subsection{Breve análise dos erros de $T$ e $q$ e das estimativas de $H$ e $L E$}

A Figura 2 mostra a variação dos erros médios de $T$ e $q$ para as escalas determinadas. Nota-se a tendência de diminuição dos erros de $T$ e $q$ à medida que o método é executado para escala mensal. No entanto, é possível notar um segundo mínimo na escala temporal de 15 dias, evidenciando as menores diferenças entre $T$ e $q$ observados e estimados para essas duas escalas. Porém, a média mensal apresenta os menores erros. Essa avaliação nos dá um indício da menor variabilidade de $T$ e $q$ ocorrendo na média mensal o que induz a pensar que as melhores estimativas de $H$ e $L E$ também encontram-se nessa escala. Entretanto, ao observar a Figura 3 verifica-se tendência oposta entre os erros relativos de $H$ e $L E$, em que o primeiro apresenta maior variabilidade exibindo um mínimo na escala temporal de 20 dias,enquanto que $L E$ mostra menor variação e exibe um máximo em 20 dias. Isso é o contrário do esperado, em que as melhores estimativas deveriam estar próximas da escala mensal, uma vez que o método sendo executado numa escala próxima da diária, os termos advectivos de $T$ e $q$ se tornam importantes. Somado a isso, quando analisa-se os fluxos separadamente, se observa que a variação não converge com a dos erros de $T$ e $q$ (Figura 2). $L E$ além de apresentar os maiores erros, apresenta uma leve tendência de aumento da escala de 5 a 20 dias, com posterior queda em 30 dias. Por outro lado, $H$ apresenta uma tendência de redução mais intensa até os 20 dias, com posterior elevação em 30 dias. Até o momento não foi encontrado uma explicação para essas variações dos erros relativos dos fluxos de $H$ e $L E$. Talvez isso seja consequência da análise ter sido feita mês a mês, considerando os últimos dias de cada mês, e não para a toda a série.

\section{Conclusões}

De acordo com os resultados: os erros de $T$ e $q$ são menores em 15 e 30 dias. Enquanto, que para os fluxos de $H$ e $L E$ os erros foram menores para a escalas de 20 e 30 dias, respectivamente.

\section{Referências}

Bastiaanssen, W., Menenti, M., Feddes, R., Holtslag, A. (1998). A remote sensing surface energy balance algorithm for land (sebal). 1. formulation. Journal of hydrology, 212, 198-212.

Brutsaert, W. (2013). Evaporation into the atmosphere: the-

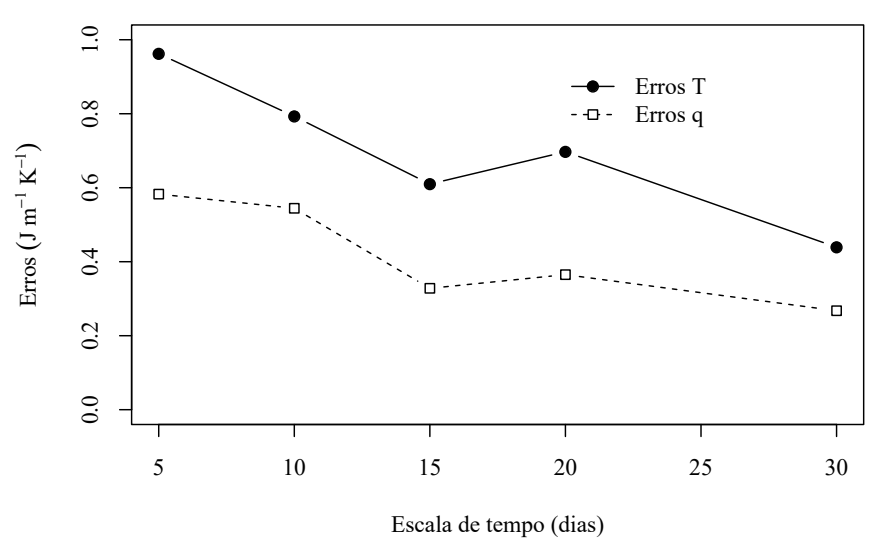

Figura 2: Erros médios de $T$ e $q$ obtidos após a execução do método para as escalas temporais médias: 5,10,15,20 e 30 dias gerados a partir dos dados de Santa Maria, RS no período entre novembro de 2013 a novembro de 2014

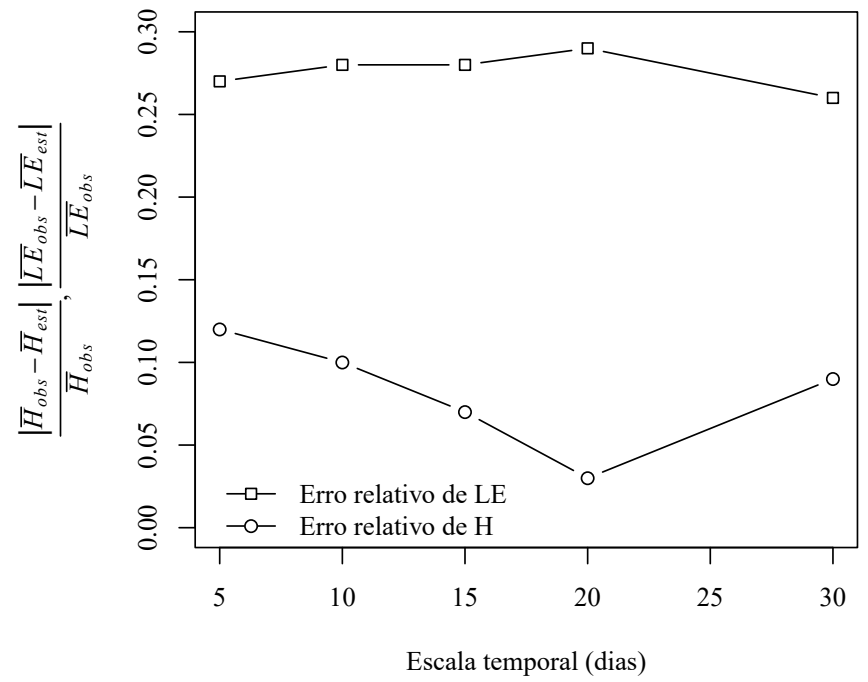

Figura 3: Erros relativos de $H$ e $L E$ para as escalas temporais médias: $5,10,15,20$ e 30 dias gerados a partir dos dados de Santa Maria, RS no período entre novembro de 2013 a novembro de 2014

ory, history and applications, vol 1. Springer Science \& Business Media.

Fitzjarrald, D. R., Acevedo, O. C., Moore, K. E. (2001). Climatic consequences of leaf presence in the eastern united states. Journal of Climate, 14(4), 598-614.

Foken, T., Gckede, M., Mauder, M., Mahrt, L., Amiro, B., Munger, J. (2004). Post-eld data quality control. .

Moncrieff, J. B., Massheder, J., De Bruin, H., Elbers, J., Friborg, T., Heusinkveld, B., Kabat, P., Scott, S., Søgaard, H., Verhoef, A. (1997). A system to measure 
surface fluxes of momentum, sensible heat, water vapour and carbon dioxide. Journal of Hydrology, 188, 589-611.

Nakai, T., Shimoyama, K. (2012). Ultrasonic anemometer angle of attack errors under turbulent conditions. Agricultural and forest meteorology, 162, 14-26.

Sorbjan, Z. (2007). A numerical study of daily transitions in the convective boundary layer. Boundary-layer meteorology, 123(3), 365-383.

Stull, R. B. (1988). An introduction to boundary layer meteorology, vol 13. Springer Science \& Business Media.

Veldman, J. W., Overbeck, G. E., Negreiros, D., Mahy, G., Le Stradic, S., Fernandes, G. W., Durigan, G., Buisson, E., Putz, F. E., Bond, W. J. (2015). Tyranny of trees in grassy biomes. Science (New York, NY), 347(6221), 484.

Webb, E., Pearman, G., Leuning, R., et al. (1980). Correction of flux measurements for density effects due to heat and water vapour transfer. Quarterly Journal of the Royal Meteorological Society, 106(447), 85-100.

Wilczak, J. M., Oncley, S. P., Stage, S. A. (2001). Sonic anemometer tilt correction algorithms. Boundary-Layer Meteorology, 99(1), 127-150. 